\title{
Progress on the Development of DEPFET Based SDD and MPD Detectors
}

\author{
J.Treis*, K.Heinzinger**, K.Hermenau**, S.Herrmann***, T.Lauf***, P.Lechner**, G.Lutz**, \\ P.Majewski**, M.Porro***, R.H.Richter***, G.Schaller***, F.Schopper***, H.Soltau**, \\ L.Strüder***, G.de Vita*** \\ *PNDetector GmbH, Emil-Nolde-Str.10, D-81735 München, Germany \\ **PNSensor GmbH, Römerstr. 28, D-80803 München, Germany \\ ***MPI Halbleiterlabor, Otto-Hahn-Ring 6, D-81739 München, Germany
}

For many years it has been one of the top priorities in radiation detector development to reduce the detector input capacitance to the lowest possible value, as it affects the way all kinds of noise impact the spectral resolution. Minimizing the capacitance of the readout node allows to improve the energy resolution for given timing or, correspondingly, drastically increase the optimum shaping time constant with unaltered spectral performance. The combined detector-amplifier structure DEPFET [1] (Depleted P-channel FET) provides an ultra-low input capacitance, allowing for X-ray detection with near Fano-limited energy resolution at high readout speeds.

Being the most sophisticated application of the principle of sidewards depletion, DEPFETs increasingly find application as basic building blocks for radiation detectors in a large variety of scientific fields, e.g. as focal plane detector for space borne X-ray telescopes [2]. Fig. 1 shows a cutaway of a circularly shaped standard DEPFET cell, consisting of a traditional p-channel FET integrated onto a fully depleted n-type silicon bulk. By means of implantation profiles and biasing voltages, a potential minimum for electrons is created, which is located directly beneath the transistor channel. Bulk-generated charge is collected here, where their presence modulates the PMOS channel conductivity. In this way, the transistor current becomes a function of the charge in the potential minimum (the so-called internal gate), and sensing the channel conductivity by means of e.g. a source follower or transimpedance amplifiers, gives a measure for the collected charge. By bringing the charge cloud that close to the sensitive readout node, an equivalent readout capacitance as low as $40 \mathrm{fF}$ can be achieved [3].

DEPFETs can be read out just like a conventional SDD in a time-continuous way, where the arrival of the charge is sensed. But as the charge remains confined in the internal gate, DEPFETs can also be read out in an on-demand fashion, where the step size is sensed while the charge is removed from the internal gate using an additional n-channel ClearFET. The latter readout mode can be used to build large, area and power efficient integrating type imaging detectors. DEPFET based readout nodes can also be combined with a drift ring structure to form a so-called Macropixel (see Fig. 1). Similar to an SDD detector, shape and size of the Macropixels can be arbitrarily adapted to the experimental requirements [2], sensor devices with large sensitive areas and optimized pixel geometries can be generated. Such a device combined the traditional advantages of SDDs, like scalability and optimized QE, with the advantages of the DEPFET cell, e.g. low input capacitance and charge storage capability. Devices recently operated show an energy resolution of $125 \mathrm{eV}$ FWHM@5.9. keV for a sensitive area of $1.9 \times 1.9 \mathrm{~cm}^{2}$ at a framerate of $6 \mathrm{kHz}$ (Fig. 2).

In previous years the development focused on the development of large area MPD sensors. Recently, single cell SDD devices utilizing DEPFET based readout nodes have been developed to make the 
advantage of the reduced input capacitance become adaptive in high rate X-ray detection. These devices, which are currently under investigation, are expected to translate the decrease in input capacitance into increased rate capability. Both detector types, MPDs and DEPFET SDDs, are promising new opportunities for ultrafast low noise material analysis.

\section{References}

[1] J. Treis et al., IEEE TNS, 52 (Iss 4), (2005) p. 1083.

[2] J. Treis et al., JINST, 4 (2009) p. 03012.

[3] M. Porro et al., IEEE TNS, 53 (Iss. 1), (2006) p. 401.
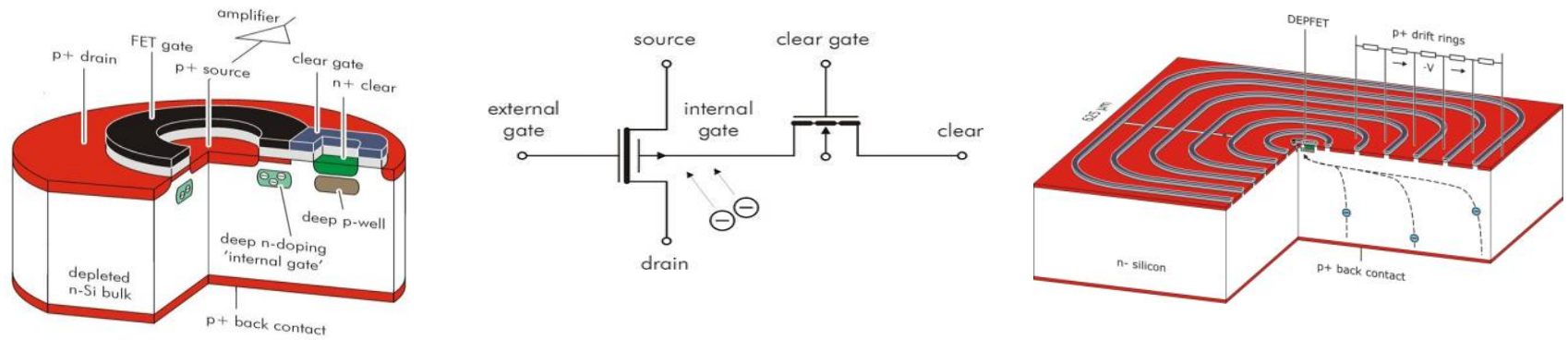

FIG. 1: Cutaway (left) of a standard DEPFET cell. The charge is stored in a dedicated potential minimum for charge directly underneath the external gate. The stored charge can be removed by the additional n-channel ClearFET. As the reset is complete, the DEPFET is a kTC noise free device. The circuit schematic (middle) shows the basic functionality: a P-channel FET with two gates: an external and an internal gate, where the latter one is contacted with an additional N-channel FET. The DEPFET cell can replace the traditional readout anode of an SDD to form a Macropixel (right).
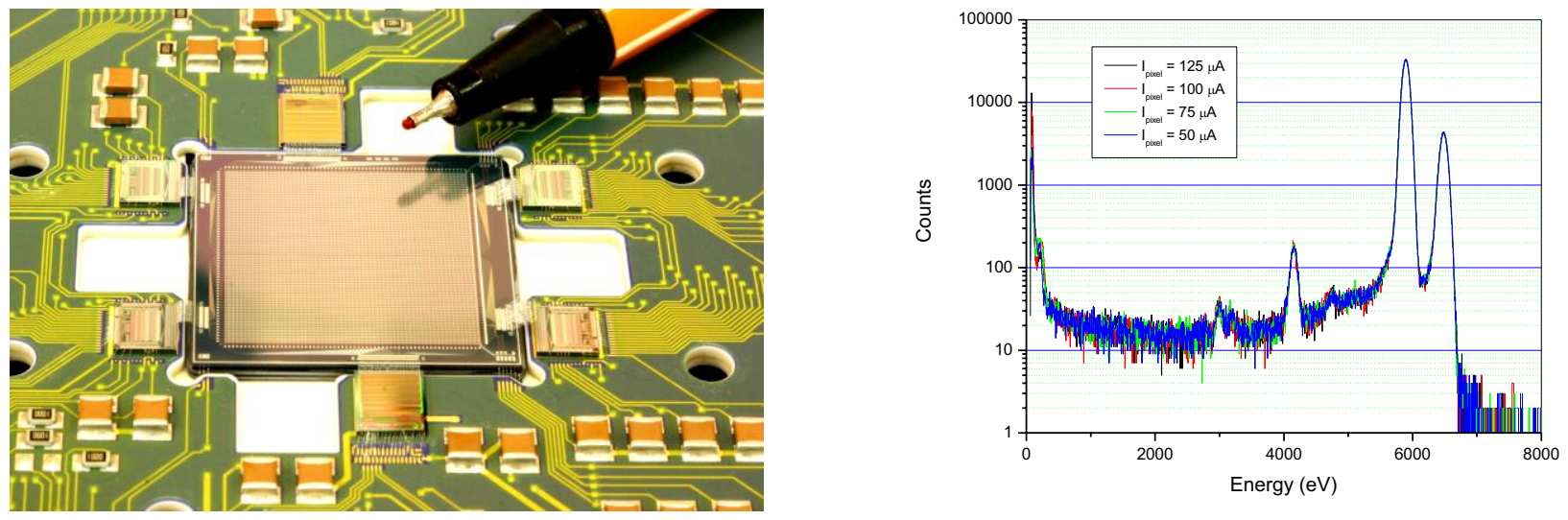

FIG. 2: Measurements from a Macropixel detector (MPD) device. Left: Photo of the 64 x 64 pixel array with $300 \times 300 \mu \mathrm{m}^{2}$ pixel size and an overall sensitive area of $19 \times 19 \mathrm{~mm}^{2}$. Right: Spectra measured for various pixel operating currents. The devices show a nearly constant energy resolution below 126 eV FWHM@ @ $5.9 \mathrm{keV}$ for a framerate of $7 \mathrm{kHz}$. 